\title{
Aloof Beam Plasmons in Silver Nanoparticles
}

\author{
Brian Zutter ${ }^{1}$, Matthew Mecklenburg ${ }^{2}$, and B. C. Regan ${ }^{1}$ \\ 1. Department of Physics and Astronomy \& California NanoSystems Institute, University of California, \\ Los Angeles, CA, USA \\ 2. Center for Electron Microscopy and Microanalysis, University of Southern California, Los Angeles, \\ CA, USA
}

Plasmon resonances in silver nanoparticles have significant applications in biology, nanophotonics, and solar energy harvesting [1]. We use electron energy loss spectroscopy (EELS) to spatially map the plasmon signal in silver nanoparticles between $7 \mathrm{~nm}$ and $40 \mathrm{~nm}$ in diameter. We observe a strong aloof beam effect where the electron beam generates plasmons when located over $4 \mathrm{~nm}$ away from the physical edge of the particle. Surface plasmon and bulk plasmon signals have previously been measured with an energy-monochromated scanning transmission electron microscope (STEM) with a zero-loss peak (ZLP) full-width at half maximum (FWHM) of 0.15 eV[1]. Our microscope, with a ZLP FWHM of $0.7 \mathrm{eV}$, lacks the energy resolution to differentiate the surface and bulk plasmons.

We measured the spatial dependence of the silver plasmon signal with a STEM (JEOL JEM-2100F) operating at $80 \mathrm{kV}$ and an EELS spectrometer (Gatan Quantum SE) set to a dispersion of 10 $\mathrm{meV} /$ channel. Spectrum images of silver nanoparticles supported on a silicon nitride membrane were acquired at room temperature. Figure 1 shows silver plasmon energy maps made by taking the energy difference between two peaks: the ZLP (Gaussian fit between $-0.45 \mathrm{eV}$ and $0.45 \mathrm{eV}$ ) and the silver plasmon peak (1.97 eV and $4.10 \mathrm{eV}$ ). The plasmon fitting window is large enough to encompass both the (unresolved) surface and bulk plasmons, which occur at $3.3 \mathrm{eV}$ and $3.85 \mathrm{eV}$ respectively [1].

Spatial maps of the plasmon energy (Figure 1) show lower energies near the particle edge and higher energies toward the center, as expected due to the changing ratio of surface relative to bulk plasmon generation. Surprisingly, the amplitude of the plasmon signal reaches its maximum outside of the edge of the particle. Evidently plasmons are more efficiently excited in silver nanoparticles by an aloof beam.

To more clearly show the aloof beam effect, we generate line profiles (3 pixel integration width) along diameters of a nanoparticle using the dark field (DF), ZLP amplitude, plasmon energy, and plasmon amplitude images. These profiles are plotted together in Figure 2. The FWHM (external to the nanoparticle) of the plasmon amplitude signal is $28 \mathrm{~nm}$, while the FWHM of the DF signal is only 20 $\mathrm{nm}$. The decrease in plasmon intensity toward the interior of the particle corresponds to a large decrease in the ZLP intensity, suggesting that higher order inelastic scattering events are becoming important. Aloof beam plasmon excitations have been observed in other metals [3], but silver nanoparticles are unusual in that a plasmon is more likely to be excited by an aloof electron beam than an incident beam.

\section{References:}

[1] J A Scholl, A L Koh and J A Dionne Nature 483 (2012), p. 421.

[2] H Raether in "Surface Plasmons on Smooth and Rough Surfaces and on Gratings", (Springer-Verlag, New York,1988).

[3] G Abajo, Reviews of Modern Physics 82 (2010), p. 209. 
[4] This work was supported by FAME, one of six centers of STARnet, a Semiconductor Research Corporation program sponsored by MARCO and DARPA. Data presented were acquired at the Center for Electron Microscopy and Microanalysis at the University of Southern California.
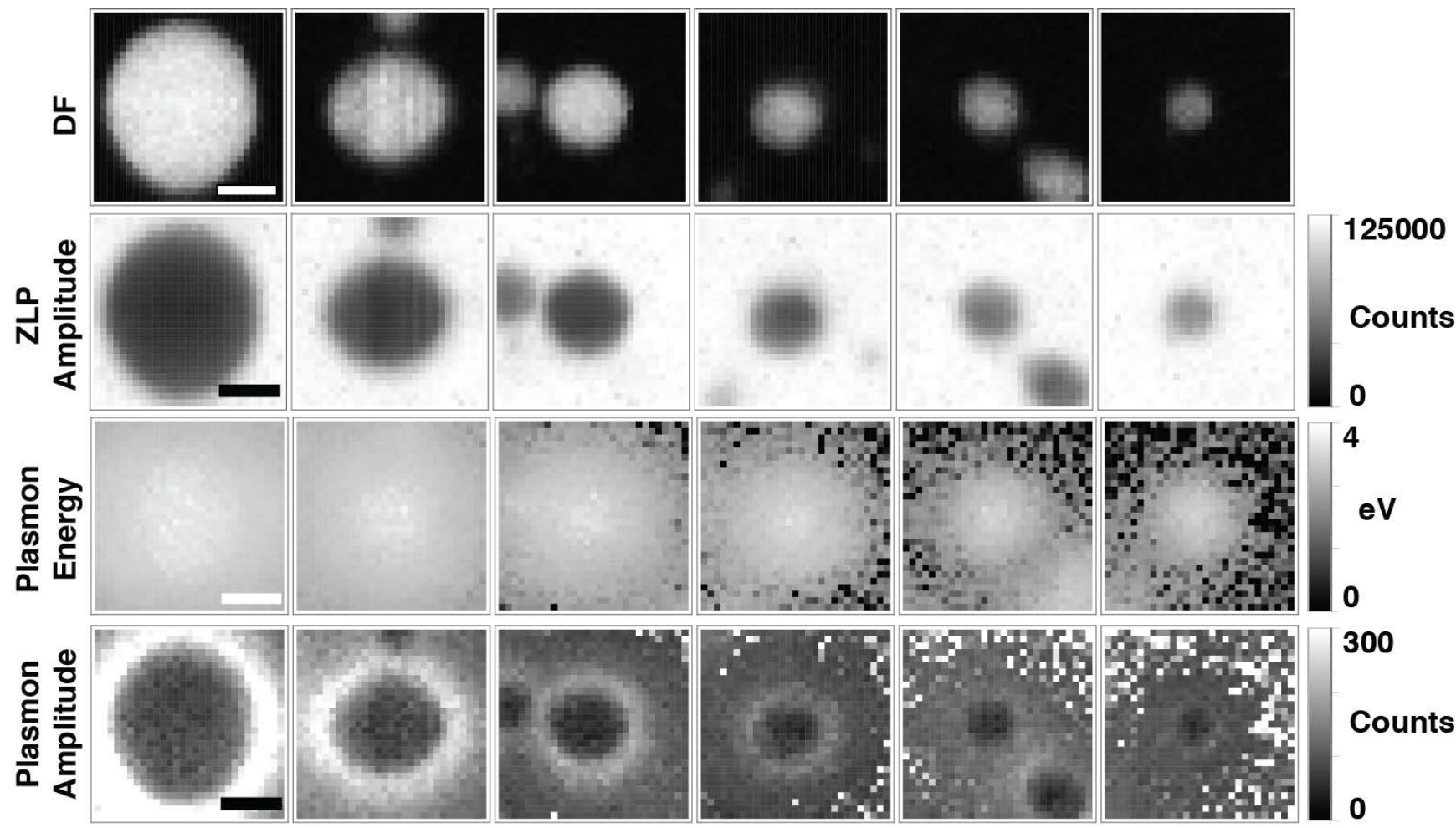

Figure 1. (first row) DF maps of 6 spherical silver particles with approximate diameters of 37, 26, 20, 15, 13, and $9 \mathrm{~nm}$. (second row) Corresponding zero loss peak (ZLP) amplitude maps. (third row) Corresponding plasmon energy maps. (fourth row) Corresponding plasmon amplitude maps. Scale bar is $15 \mathrm{~nm}$.

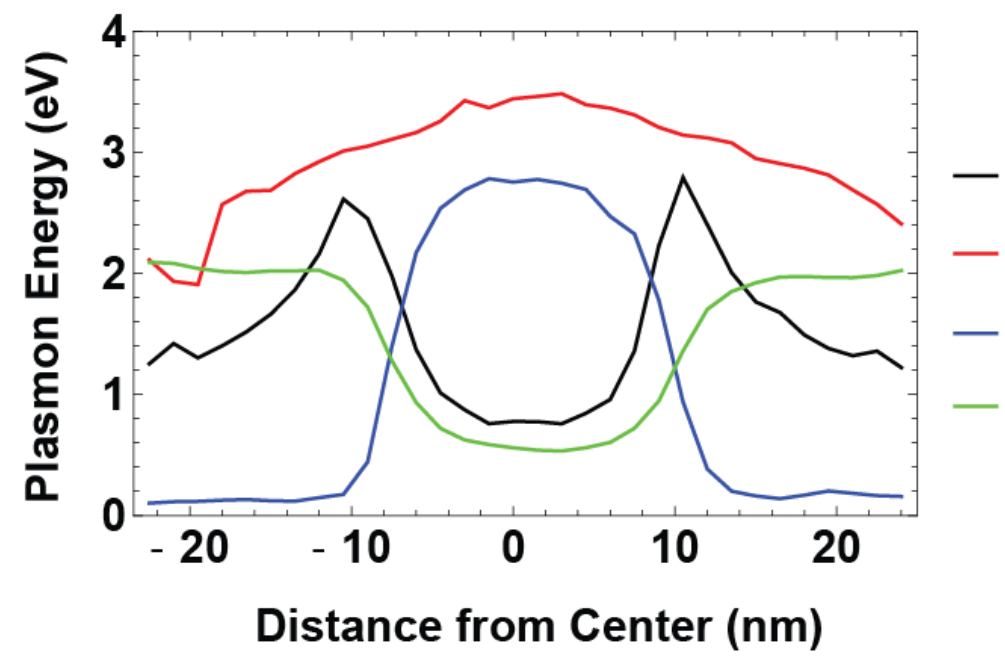

\section{Plasmon Amplitude}

Plasmon Energy

DF

\section{ZLP Amplitude}

Figure 2. Plasmon center (red), amplitude (black), DF (blue), and ZLP amplitude (green) signals in the vicinity of a $20 \mathrm{~nm}$ diameter silver nanoparticle (third column from the left of Figure 1). The plasmon amplitude and DF signals have been normalized to have a maximum amplitude of $2.75 \mathrm{eV}$, and the ZLP signal has been normalized to have a maximum amplitude of $2.09 \mathrm{eV}$. 